\title{
Inhaled phosphodiesterase type 5 inhibitors restore chloride transport in cystic fibrosis mice
}

\author{
B. Lubamba*, J. Lebacq ${ }^{*}$, G. Reychler ${ }^{\top,+}$, E. Marbaix ${ }^{\S}$, P. Wallemacq*, \\ P. Lebecque and T. Leal*,f
}

ABSTRACT: Sildenafil and vardenafil, two selective inhibitors of phosphodiesterase type 5 (PDE5) are able, when applied by intraperitoneal injection, to activate chloride transport in cystic fibrosis (CF) mice homozygous for the F508del mutation. Oral treatment with the drugs may be associated with adverse haemodynamic effects. We hypothesised that inhaled PDE5 inhibitors are able to restore ion transport in F508del CF airway epithelium.

We developed a restraint-free mouse chamber for inhalation studies. PDE5 inhibitors were nebulised for $15 \mathrm{~min}$ at concentrations adjusted from recommended therapeutic oral doses for male erectile dysfunction. We measured in vivo nasal transepithelial potential difference $1 \mathrm{~h}$ after a single inhalation of sildenafil, vardenafil or tadalafil in F508del CF and normal homozygous mice.

After nebulisation with the drugs in F508del mice, chloride transport, evaluated by perfusing the nasal mucosa with chloride-free buffer containing amiloride followed by forskolin, was normalised; the forskolin response was increased, with the largest values being observed with tadalafil and intermediate values with vardenafil. No detectable effect was observed on sodium conductance.

Our results confirm the role of PDE5 inhibitors in restoring chloride transport function of F508del CF transmembrane conductance regulator protein and highlight the potential of inhaled sildenafil, vardenafil and tadalafil as a therapy for CF.

KEYWORDS: Cystic fibrosis, inhalation, nasal potential difference, sildenafil, tadalafil, vardenafil

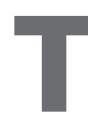
he steadily growing understanding of molecular and biological mechanisms underlying cystic fibrosis (CF) lung disease has resulted in exciting new targets for treatment. Fundamental research focusing on correcting F508del mutant protein can be considered as disease-modifying approaches and would be beneficial for most CF patients. Indeed, the F508del mutation, a misfolded protein that fails to escape the endoplasmic reticulum [1], represents the most common $\mathrm{CF}$ transmembrane conductance regulator (CFTR) mutation worldwide.

Inhalation drug therapy has several potential advantages over oral and intravenous routes, including rapid onset of pharmacological action, minimised systemic adverse effects and reduced effective drug doses compared to the same drug delivered orally [2]. Nebulisation of topically active drugs has become increasingly common for treatment in CF [3]. Novel drug formulations acting through distinct mechanisms, including restoration of airway surface liquid or mucus, anti-inflammatory or anti-infective agents, and immunosuppressive agents, have been currently tested by inhalation therapy in CF [4].

Sildenafil, vardenafil and tadalafil are highly selective inhibitors of cyclic guanosine monophosphatedependent phosphodiesterase type 5 (PDE5) [5] commonly used for improving erectile dysfunction. Moreover, oral therapy with sildenafil promotes nitric oxide-mediated pulmonary vasodilation with a dose-dependent decrease of pulmonary artery pressure and vascular resistance [6]. These features provide the rationale behind its use for the treatment of pulmonary arterial

\section{AFFILIATIONS}

*Louvain Centre for Toxicology and Applied Pharmacology,

\#Cell Physiology,

-Paediatric Pulmonology,

+Physical Medicine and

Rehabilitation,

${ }^{\S}$ Pathology, and

${ }^{f}$ Center for Human Genetics,

Université Catholique de Louvain,

Brussels, Belgium.

CORRESPONDENCE

T. Leal

Dept of Clinical Chemistry

St Luc University Hospital

10 Av Hippocrate

B-1200 Brussels

Belgium

E-mail: teresinha.leal@uclouvain.be

Received:

Jan 262010

Accepted after revision:

May 302010

First published online:

June 182010 
hypertension. We have shown previously [7] that intraperitoneal injection of sildenafil and vardenafil to F508del CF mice rescues CFTR-dependent chloride transport. However, systemic haemodynamics, including flushing, headache and other cardiovascular effects $[8,9]$, could compromise their potential use in CF. Therefore, we hypothesised that in vivo nebulisation of PDE5 inhibitors restore F508del CFTR chloride transport. In the present study, we developed a low-stress mouse chamber for inhalation studies and sought to investigate the efficacy of inhaled PDE5 inhibitors on ion transport across the nasal mucosa of F508del CF mice. Our results provide clear evidence that inhalation of PDE5 inhibitors restores chloride transport across the respiratory epithelium of F508del CF mice.

\section{METHODS}

\section{Animal model}

Young adult CF mice homozygous for the F508del CFTR mutation in the 129/FVB outbred background ( $\left.\mathrm{cttr}^{\mathrm{tm}^{\mathrm{t}} 1 \mathrm{Eur}}\right)$ [10] and their normal homozygous wild-type littermates were studied. Mouse age ranged $4-5$ months and their weights ranged 20-30 g. Animals were housed at the Animal Care Facility (Université Catholique de Louvain, Brussels, Belgium) following the recommendations of the Federation of European Laboratory Animal Science Associations [11]. To prevent intestinal obstruction in CF animals, Movicol $\left(55.24 \mathrm{~g} \cdot \mathrm{L}^{-1}\right.$; Norgine, Heverlee, Belgium) was administered in acidified drinking water. The mice were genotyped at 21 days of age using TaqMan quantitative PCR (Applied Biosystems, Carlsbad, CA, USA), as previously described [12]. These studies and procedures were approved by the local Ethics Committee for Animal Welfare (Université Catholique de Louvain) and conformed to the European Community regulations for animal use in research (CEE no. 86/609).

\section{Preparation of PDE5 inhibitors}

Stock nebuliser solutions were prepared within the limit of water solubility for each PDE5 inhibitor, i.e. $3.5 \mathrm{mg} \cdot \mathrm{mL}^{-1}$ for sildenafil citrate, $1.1 \mathrm{mg} \cdot \mathrm{mL}^{-1}$ for vardenafil and $0.002 \mathrm{mg} \cdot \mathrm{mL}^{-1}$ for tadalafil. Nebuliser solutions were used at concentrations adjusted from recommended initial oral doses for improving erectile dysfunction, i.e. $0.7 \mathrm{mg} \cdot \mathrm{kg}^{-1}$ sildenafil and $0.14 \mathrm{mg} \cdot \mathrm{kg}^{-1}$ vardenafil or tadalafil. For each animal ( $\sim 25 \mathrm{~g}$ body weight), the required amount of drugs corresponding to $0.0175 \mathrm{mg}$ sildenafil citrate, $0.0035 \mathrm{mg}$ vardenafil hydrochloride or tadalafil was formulated in $3 \mathrm{~mL}$ saline, stored at $4{ }^{\circ} \mathrm{C}$ and used within 3 days of preparation. Saline was nebulised in placebo control experiments. Sildenafil citrate was obtained from Pfizer (Sandwich, UK), vardenafil from Bayer (West Haven, Germany) and tadalafil from Lilly (Brussels, Belgium).

\section{Nebulisation}

A small, transparent mouse chamber for drug inhalation studies was designed for this study. The inhalation chamber consists in a cylindrical polymethylmethacrylate box with an expiratory gate, a floor area of $113 \mathrm{~cm}^{2}$ and a volume of $960 \mathrm{~cm}^{3}$; it was designed to accommodate a single nonrestrained, conscious and spontaneously breathing mouse. A nebuliser with standardised aerodynamic properties (Sidestream; Medic-Aid, Bognor Regis, UK), driven by its own compressor (Portaneb; Medic-Aid), was connected to the top cover of the inhalation chamber. Nebuliser experiments were conducted with the reservoir filled with $3 \mathrm{~mL}$ of PDE5 inhibitor solution. Experiments were performed under dynamic conditions, the airflow being introduced continuously and vertically at the top of the chamber. Nebuliser exposure lasted for $\sim 15 \mathrm{~min}$. At the end of the exposure, the animals remained in the chamber for $5 \mathrm{~min}$ and then returned to their cages until nasal potential difference (PD) measurements were performed.

\section{Nasal PD measurements}

Nasal PD, measured as previously described [7, 13, 14], was undertaken at different time points, as indicated, after a single nebuliser exposure to PDE5 inhibitors. Transepithelial sodium conductance was evaluated by the most negative baseline value (PDmax) and the amiloride response, evaluated by the change in PDmax after perfusion with Ringer's saline solution containing $10^{-4} \mathrm{M}$ amiloride. Chloride conductance (SumCl) was evaluated by nasal perfusions with a chloride-free solution of sodium gluconate containing amiloride, followed by addition of $10^{-5} \mathrm{M}$ forskolin, an adenylate cyclase agonist. Forskolin response was evaluated by the fractional component of the global chloride transport, and corresponded to the changes after perfusion of the nasal mucosa with $10^{-5} \mathrm{M}$ forskolin. To verify the proportion of respiratory ciliated tissue at the site of nasal PD measurement, the nasal mucosa of wildtype nontreated animals was excised at the end of nasal PD experiments and tissue was fixed in $4 \%$ buffered paraformaldehyde for histological studies. Sections processed at $5 \mu \mathrm{m}$ thickness for light microscopy were stained with haematoxylin and eosin.

\section{Histological control of the site of nasal PD measurement}

Differences in the distribution of types of epithelia across the anatomical regions of the mouse nasal cavity were observed; these regional differences were not influenced by the $\mathrm{CF}$ genotype (data not shown). From the vestibulum nasi to the mid-proximal portion along the dorsal nasal concha (supplementary Figure 1A), the epithelium was predominantly olfactory (supplementary Figure 1B), with small regions of lightly keratinised squamous epithelium restricted to the nostril opening regions (supplementary Figure 1C), and transitional epithelium lining the lateral wall (supplementary Figure 1D). Proceeding caudally to the middle nasal concha, the phylogenetic equivalent of the human middle turbinate [15], progressive transition from olfactory to respiratory ciliated epithelia was found (supplementary Figure 1E). The nasal PD catheter was usually located at 4-6 $\mathrm{mm}$ from the nostril in the middle nasal concha. When, at the end of nasal PD measurements, a focal nasal lesion $(\sim 0.25 \mathrm{~mm}$; corresponding to the outside diameter of the nasal PD probe) was intentionally produced by exerting light pressure of the probe against the distal wall of the middle concha, a respiratory epithelium with ciliated cells was found at this site (fig. 1a). In the posterior region lining the perpendicular plate of the ethmoidal bone and corresponding to the ethmoidal concha, the phylogenetic equivalent of the human superior turbinate [15], mostly ciliated respiratory epithelium was found (fig. 1b). In the most distal portions of the nasal cavity, close to the olfactory bulb, regions with predominant olfactory epithelium were also found. 


\section{Statistics}

Descriptive statistics (mean \pm SEM) and tests of statistical significance were performed using the SAS-JMP software (SAS Institute, Cary, NC, USA). The number of mice was selected based on sample size calculations when setting a two sided $\alpha$ level at 0.05 and a (1- $\beta$ ) level at 0.8 . Between-group comparisons were evaluated using one-way ANOVA. Post hoc comparisons were made using unpaired t-tests or Tukey-Kramer honestly significant difference tests for two or more $x$ levels, as needed. The null hypothesis was rejected at p-values $<0.05$.

\section{RESULTS}

\section{Baseline and stimulated PD values in nontreated mice}

The nasal PD of the CF mouse is very different from that of the wild-type mouse. In CF mice [7, 14], as in CF patients [16, 17],
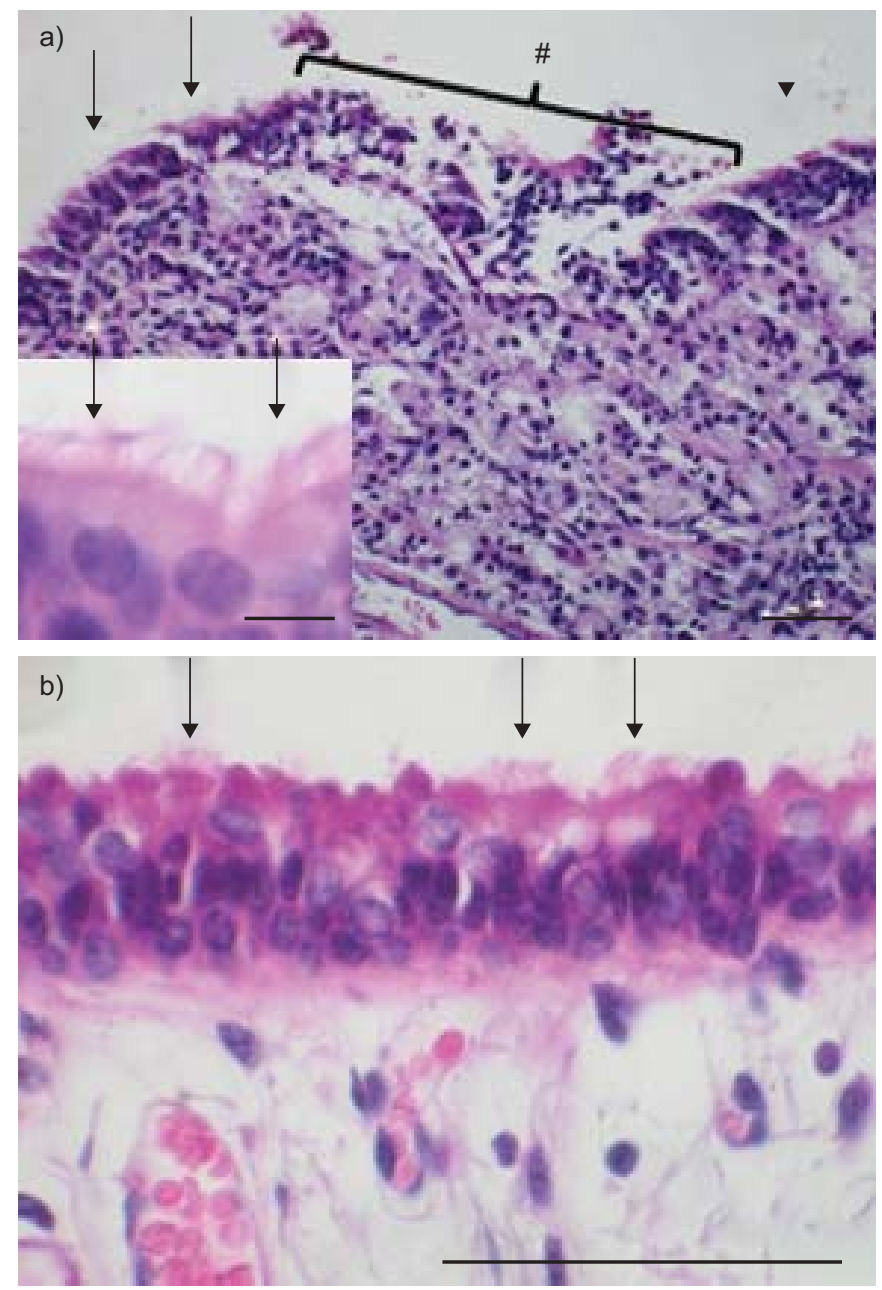

FIGURE 1. Light micrographs of the surface epithelia lining the nasal cavity of a wild-type mouse. Tissue sections stained with haematoxylin and eosin. a) Region of the middle nasal concha, where the nasal potential difference (PD) probe is usually located, showing transition from olfactory to respiratory epithelium containing numerous ciliated cells (inset; scale bar $=10 \mu \mathrm{m}$ ). Focal nasal lesion (as indicated $(\#)$ ) produced by the PD probe at the site of nasal PD measurement. Scale bar $=50 \mu \mathrm{m}$. b) Respiratory, pseudostratified, ciliated columnar epithelium lining the mid-distal portion of the septal wall of the middle concha and the midproximal region of the ethmoidal concha. Arrowheads identify olfactory epithelium; arrows identify respiratory epithelium with ciliated cells. Scale bar $=50 \mu \mathrm{m}$. the nasal PD is characterised by increased sodium conductance and reduced chloride conductance across the epithelium. Baseline hyperpolarisation (i.e. more negative basal values) and increased depolarising amiloride response, both reflecting increased epithelial sodium channel (EnaC)-dependent sodium transport activity, were found in the nasal mucosa of the F508del mouse (fig. 2). In addition, cumulated voltage changes after nasal perfusion with chloride-free buffer and with forskolin reliably discriminated between CF and wild-type animals. As illustrated in figure 2, mean SumCl in F508del mutant animals was reduced to approximately one-third of that found in controls.

\section{Nebulisation treatment}

Nebuliser experiments were performed in nonanaesthetised, spontaneously breathing animals, at room temperature, in a quiet exposure environment at the animal house, under specific pathogen-free conditions [11] with respect to clean air supply, noise, vibration, lighting and freedom of movement.

Nebuliser drug and saline treatment was well tolerated and no adverse effects were observed.

Inhalation of saline, used as placebo, did not influence any nasal PD value. Indeed, irrespective of the genotype, mean nasal PD parameters, recorded $1 \mathrm{~h}$ after inhaled saline, were similar to those monitored in the corresponding nontreated animals (data from treated wild-type group not shown in fig. 3).

\section{Aerosolised sildenafil and vardenafil}

In our previously published work [7], we have shown that in vivo intraperitoneal administration of therapeutic doses of sildenafil and vardenafil to F508del CF mice was able to rescue CFTR-dependent chloride transport across the nasal mucosa. In this work, we tested, in the same CF mouse model, the potential of the inhaled drugs. Mean SumCl values obtained from $\mathrm{CF}$

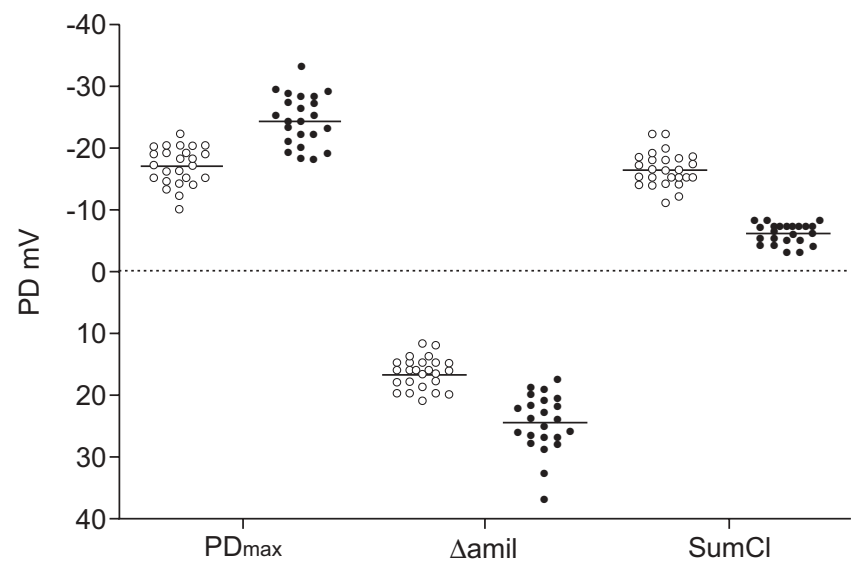

FIGURE 2. Maximal baseline difference (PD) values (PDmax), amiloride response ( $\Delta$ amil), and chloride transport (SumCl) in nontreated wild-type homozygous control $(O ; n=27)$ and F508del mice $(-n=26)$. $\Delta$ amil was evaluated by changes in nasal PD after perfusion with basal isotonic saline solution containing $10^{-4} \mathrm{M}$ amiloride. Chloride transport was evaluated by the cumulative changes in nasal PD after perfusion with chloride-free solution in the presence of amiloride, followed by addition of $10^{-5} \mathrm{M}$ forskolin. A p-value $<0.0001$ was obtained for comparison of the corresponding mean value $(-)$ for each of the three parameters versus that obtained for the same parameter in the wild-type group of mice. 


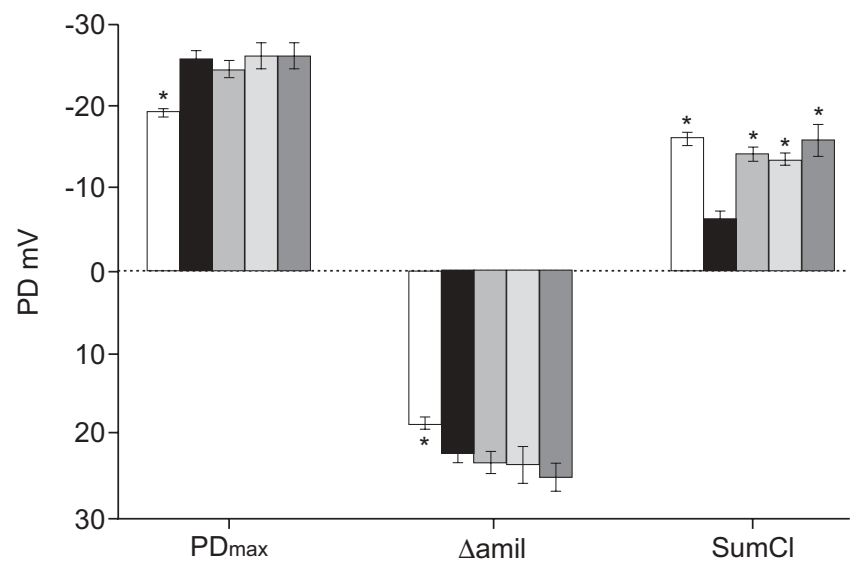

FIGURE 3. Maximal baseline difference (PD) values (PDmax), amiloride response ( $\Delta$ amil), and chloride transport (SumCl) in wild-type homozygous control $1 \mathrm{~h}$ after nebulisation with placebo $(\square)$ and in F508del mice $1 \mathrm{~h}$ after nebulisation

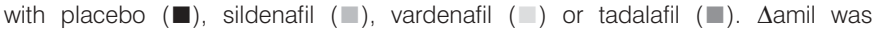
evaluated by changes in nasal PD after perfusion with Ringer's saline solution containing $10^{-4} \mathrm{M}$ amiloride. Chloride transport was evaluated by the cumulative changes in nasal PD after perfusion with chloride-free solution in the presence of amiloride followed by addition of $10^{-5} \mathrm{M}$ forskolin. Data are presented as mean \pm SEM for five to eight animals in each group. ${ }^{*}: p<0.05$ for comparison of the corresponding mean values versus those obtained for the same parameter in the placebo-treated cystic fibrosis group.

mice $1 \mathrm{~h}$ after nebulisation with sildenafil or vardenafil were significantly increased, reaching values comparable to those recorded in placebo-treated wild-type mice (fig. 3).

Sildenafil and vardenafil did not influence transepithelial sodium transport. Indeed, basal PDmax values and amiloride response were not modified $1 \mathrm{~h}$ after nebulisation with the drugs as compared to placebo (fig. 3). The correcting effect of vardenafil lasted $\geqslant 8 \mathrm{~h}$, while that of sildenafil was progressively lost over time and was completely absent $8 \mathrm{~h}$ after inhalation (fig. 4).

\section{Aerosolised tadalafil}

In our previous intraperitoneal data [7], the effect of tadalafil, a highly selective PDE5 inhibitor acting through a similar mechanism as sildenafil and vardenafil, on CFTR function could not be investigated due its extremely poor water solubility. As illustrated in figure 5, chloride transport in F508del mice was completely restored $1 \mathrm{~h}$ after nebulisation with tadalafil as compared to placebo. Mean $\mathrm{SumCl}$ values after nebulisation in CF animals reached values similar to those recorded in the placebo wild-type group (fig. 3). As for the two other PDE5 inhibitors, no effect was observed with taladafil on sodium transport (fig. 3).

\section{Comparative effects of the PDE5 inhibitors}

We next compared, in CF mice, the degree of responses to inhaled PDE5 inhibitors, by analysing the contribution of each component of the global chloride transport (i.e., the chloride diffusion potential recorded after nasal perfusions with chloride-free buffer containing amiloride, and the subsequent response to forskolin). No differences in chloride diffusion potential were detected between treated CF groups (data not

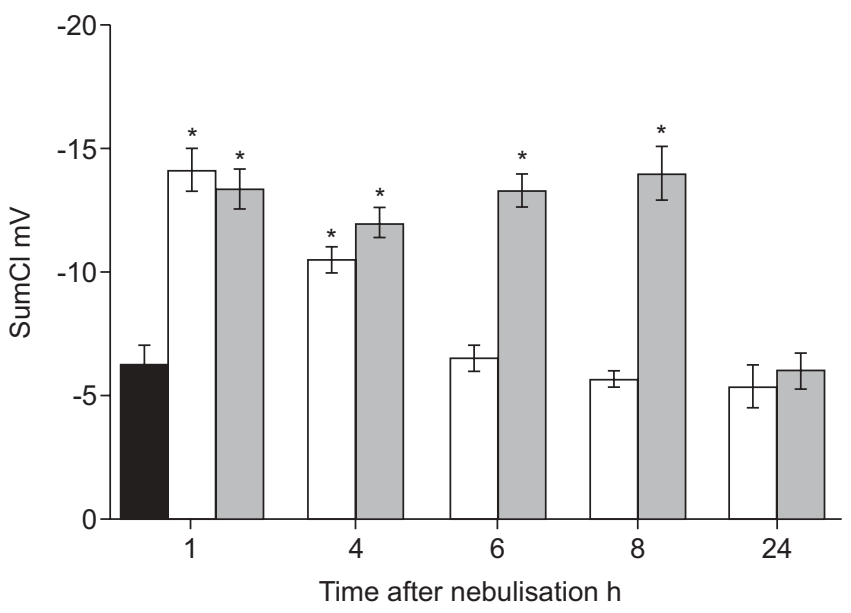

FIGURE 4. Duration of the effect of inhaled sildenafil and vardenafil on chloride transport in F508del CF mice. Nasal transepithelial potential difference (PD) was measured $1,4,6,8$ or $24 \mathrm{~h}$ after a single nebulisation with placebo (Ш), sildenafi $(\square)$ or vardenafil ( $\square$ ). Chloride transport (SumCl) was evaluated by the cumulative changes in nasal PD after perfusion with chloride-free solution in the presence of amiloride, followed by addition of $10^{-5} \mathrm{M}$ forskolin. Data are presented as mean \pm SEM for three to seven animals in each group. ${ }^{*}: p<0.05$ for comparison of the corresponding mean value versus that obtained for the same parameter in the placebo-treated group of mice.

shown). However, differences among treated groups were observed on the forskolin response. As illustrated in figure 6, forskolin response was at least three times larger in the sildenafil-treated mice compared to the placebo group. The largest values were found in the tadalafil group and intermediate values in the vardenafil group (fig. 6).

\section{DISCUSSION}

The present work was designed to investigate whether inhalation of PDE5 inhibitors, using three drugs approved

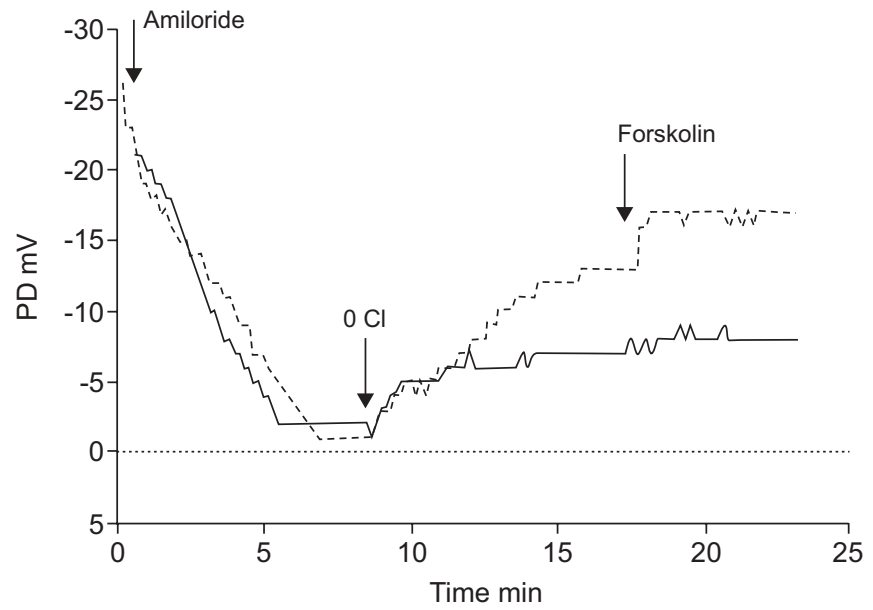

FIGURE 5. Nasal transepithelial potential difference (PD) measurements in F508del CF mice $1 \mathrm{~h}$ after nebulisation with placebo $(-)$ or tadalafil (---) Representative tracings show sequential response of the nasal mucosa to perfusion consecutively with Ringer's saline solution, Ringer's solution containing $10^{-4} \mathrm{M}$ amiloride, chloride-free solution with amiloride $(0 \mathrm{Cl})$, followed by addition of $10^{-5} \mathrm{M}$ forskolin. Arrows indicate changes of solutions. 


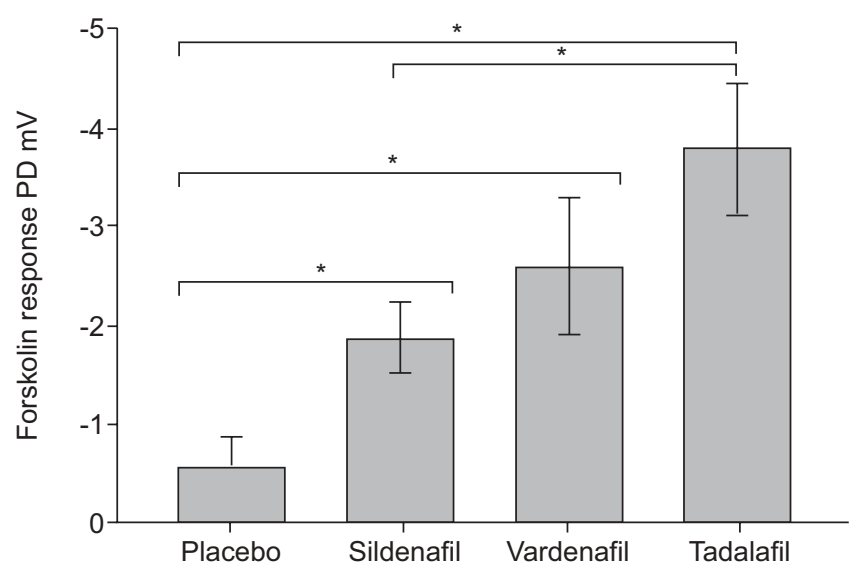

FIGURE 6. Effect of phosphodiesterase type 5 inhibitors on forskolin response in F508del CF mice. A nasal transepithelial potential difference (PD) test was performed $1 \mathrm{~h}$ after nebulisation with placebo, sildenafil, vardenafil or tadalafil in F508del CF mice. Forskolin response was evaluated by the fractional component of the global chloride transport, and corresponded to the changes observed after perfusion of the nasal mucosa with $10^{-5} \mathrm{M}$ forskolin solution. Data are presented as mean \pm SEM for five to eight animals in each group. *: two-sided $\alpha$ level of 0.05 for the corresponding parameter.

for non-CF indications, influences transepithelial ion transport defects of the F508del CF mouse. In our previously published data [7], we have demonstrated that intraperitoneal administration of therapeutic oral doses of sildenafil and vardenafil, two potent and selective PDE5 inhibitors initially developed for treatment of male erectile dysfunction, is followed by complete restoration of CFTR-dependent chloride transport defects across the nasal mucosa of F508del CF mice and that it does not involve an alternative chloride transport pathway. In the present work, we tested the three clinically used oral PDE5 inhibitors, sildenafil, vardenafil and tadalafil. We showed that their administration through a single inhalational exposure is able to restore chloride transport across the respiratory epithelium of $\Delta$ F598 CF mice and that the effect of vardenafil lasts $\geqslant 8 \mathrm{~h}$.

Respiratory delivery to mice is technically challenging due to inherent anatomical and physiological animal characteristics, and, particularly, to small animal size [18-20]. The nebuliser setup we developed in the present work comprises a wholebody immersion exposure chamber designed for a single mouse. Major advantages of a whole-body inhalation chamber over other methods of inhalation exposure for live animals, including head-only exposures, nose- or mouth-only methods, lung-only exposures, and partial-lung exposures [18-20], comprise lack of restraint or anaesthesia during experiment, and a controllable low-stress environment with respect to clean air supply, noise, vibration and lighting exposure. These practical aspects, while bringing physical comfort for the animal, minimise environmental stresses, which may have detrimental effect on data. Moreover, whole-body chambers can generate relatively uniform and stable aerosol drug concentrations throughout the exposure zone with reduced interexperiment variability [18-20]. A disadvantage of wholebody exposure chambers is the considerable loss of inhaled test drugs in the animal fur, eyes and mouth, and in the chamber walls. However, no skin, eye or mouth irritation was observed in the present study.

The ideal subject for studies relevant to man is man himself. However, the design of clinical trials can benefit from research validated in pre-clinical animal models. The nasal mucosa of our F508del mouse model [10] has proven to be valuable for studying novel therapeutic strategies that aim at activating chloride and/or sodium transport function [7, 14]. Recently, concerns on the relevance of the murine nasal mucosa as a human-equivalent model have emerged [21]. These concerns are based on the presence of differential proportions of various cell types constituting the mouse and the human nasal epithelium [22] and, subsequently, on different functional roles played by each cell population subset. Accordingly, in the mouse, the respiratory ciliated and the olfactory epithelia line $\sim 46$ and $\sim 47 \%$ of the nasal cavity, respectively, with small regional fractions being covered by squamous and transitional epithelia [22]. In contrast, in humans, respiratory epithelium lines the large majority of the nasal cavity with only $\sim 3 \%$ of the nasal surface being covered by olfactory epithelium [23]. Therefore, attention has been drawn to the issue that assessing respiratory transepithelial ion transport in the nose of mouse, as during nasal PD measurements, may possibly integrate the contribution of the olfactory neuroepithelium in the generation of bioelectrical signals [21]. We confirmed here that the nasal PD in mouse can be directed at the respiratory epithelium with high density of ciliated epithelial cells found at the site of nasal PD measurement. This finding highlights the nasal PD in mouse as a valuable tool to evaluate efficacy of fundamental pharmacological therapies in CF. As the olfactory epithelium in CF mice shows progressive, age-related morphological and functional losses [21], the changes we observed in adult CF mice after treatment with PDE5 inhibitors are not expected to reflect olfactory epithelium modifications.

Nasal PD data obtained here confirmed our previously published data [7], identified the inhalational route as a potential therapy for sildenafil and vardenafil in $C F$, and demonstrated, for the first time, that tadalafil also restores airway transepithelial chloride transport through the F508del CFTR protein. We showed here that nebulisation exposure to an amount of the three clinical available PDE5 inhibitors adjusted from oral therapeutic doses was able to stimulate forskolin-dependent chloride conductance across the respiratory epithelium of the F508del CF mouse. Distinct relative potencies among the drugs may contribute to the different degrees of forskolin response that we observed (in decreasing order: tadalafil $\geqslant$ vardenafil $>$ sildenafil). Indeed, vardenafil and tadalafil are more potent and selective than sildenafil at inhibiting PDE5 [5]. As previously reported [7], no significant effect of PDE5 inhibitors was detected on sodium transport. These data might argue against a direct reciprocal relationship between CFTR and ENaC activity. This could reflect a property of the murine nose where sodium hyperabsorption is less pronounced than in the human nose: indeed, basal PD values recorded in $\mathrm{CF}$ patients are on average substantially more negative than in F508del-CF mice $[16,17]$.

Local activation of CFTR protein function following airway deposition may be expected to contribute to the pharmacodynamic effects observed. As mice are obligate nose breathers 
[23], drug exposure in the mouth during nebulisation in a whole-body inhalation chamber model, and subsequent licking of fur and chewing drugs deposited in skin/mouth, might scarcely contribute to the bioavailability of PDE5 inhibitors during whole-body inhalation exposures. Consistent with our results, a similar CFTR-correcting effect of sildenafil has been originally shown in cultures of nasal epithelial cells harvested from CF patients and attached to an impermeable support, a configuration that allows interaction of drugs through the apical surface of epithelia [24]. It has been demonstrated recently that the inhalation route of administration for vardenafil is associated with an acceptable safety profile [25]. Apart from brief coughing on inspiration, no clinically significant changes in blood pressure or heart rate and no serious adverse events were recorded [25].

A recent study reported that sildenafil therapy in a patient with severe CF lung disease was followed by clinically significant improvements in exercise tolerance and pulmonary hypertension [26]; even though its prevalence is difficult to establish, pulmonary hypertension develops in a significant proportion of adult CF patients. It has been recognised that subclinical pulmonary hypertension correlates with the severity of the disease and is associated with increased mortality in CF [27]. However, further studies designed to examine the beneficial effect of PDE5 inhibitors on pulmonary hypertension in CF patients are needed.

Additionally, potential interest in PDE5 inhibitors to treat $\mathrm{CF}$ might be raised by the assumption that PDE5 inhibitors have a possible anti-inflammatory action [28-30]. In fact, it has been shown that application of sildenafil for $24 \mathrm{~h}$ to cultured CF bronchial epithelial cells exposed to Pseudomonas aeruginosa, a crucial pathogen responsible for the progressive loss of lung function in CF, reduced secretion of interleukin-8, a cytokine abnormally elevated in CF and responsible for neutrophil infiltrate and subsequent inflammatory cascade [29]. These data further support the view of a local airway effect of PDE5 inhibitors. A clinical trial, to be performed at our centre, aiming to investigate the effect of a single nasal topical administration of vardenafil on nasal PD measurements in CF patients homozygous for the F508deldel mutation is listed on www.clinicaltrials.gov (identifier number NCT01002534).

In the present study, we developed a low-stress setup for inhalation studies in small animals. We confirmed the potential of sildenafil and vardenafil to restore chloride transport defects of the CFTR protein, and we showed, for the first time, that tadalafil is also able to correct chloride transport defects across the nasal mucosa of F508del CF mice. The three PDE5 inhibitors, applied by inhalation, restored chloride transport across the respiratory epithelium and the effect of vardenafil lasted $\geqslant 8 \mathrm{~h}$.

\section{SUPPORT STATEMENT}

This study was supported by the Dept of Pneumology and the Fondation St Luc, St Luc University Hospital and Université Catholique de Louvain, Brussels, Belgium. Breeding pairs of $\mathrm{ctrr}^{\mathrm{tmleur}}$ (F508del (FVB/129)) mice were obtained from B. Scholte (Erasmus Medical Center Rotterdam, Rotterdam, The Netherlands) with the support of European Economic Community European Coordination Action for Research in Cystic Fibrosis EU FP6 LSHM-CT-2005-018932).

\section{STATEMENT OF INTEREST}

None declared.

\section{REFERENCES}

1 Amen N, Silvis M, Bradbury NA. Endocytic trafficking of CFTR in health and disease. J Cyst Fibros 2007; 6: 1-14.

2 Dalby R, Suman J. Inhalation therapy: technological milestones in asthma treatment. Adv Drug Deliv Rev 2003; 55: 779-791.

3 Geller DE. Aerosol antibiotics in cystic fibrosis. Respir Care 2009; 54: 658-670.

4 Cystic Fibrosis Foundation. Drug Development Pipeline. www.cff. org/treatments/pipeline Date last accessed: September 2010. Date last updated: September 27, 2010.

5 Blount MA, Beasley A, Zoraghi R, et al. Binding of tritiated sildenafil, tadalafil, or vardenafil to the phosphodiesterase-5 catalytic site displays potency, specificity, heterogeneity, and cGMP stimulation. Mol Pharmacol 2004; 66: 144-152.

6 Weimann J, Ullrich R, Hromi J, et al. Sildenafil is a pulmonary vasodilator in awake lambs with acute pulmonary hypertension. Anesthesiology 2000; 92: 1702-1712.

7 Lubamba B, Lecourt H, Lebacq J, et al. Preclinical evidence that sildenafil and vardenafil activate chloride transport in cystic fibrosis. Am J Respir Crit Care Med 2008; 177: 506-515.

8 Doggrell SA. Comparison of clinical trials with sildenafil, vardenafil and tadalafil in erectile dysfunction. Expert Opin Pharmacother 2005; 6: 75-84.

9 Bartolome SD, Channick RN. Sildenafil for pulmonary arterial hypertension. Future Cardiol 2006; 2: 137-143.

10 van Doorninck JH, French PJ, Verbeek E, et al. A mouse model for the cystic fibrosis delta F508 mutation. EMBO J 1995; 14 4403-4411.

11 Nicklas W, Baneux P, Boot R, et al. Recommendations for the health monitoring of rodent and rabbit colonies in breeding and experimental units. Lab Anim 2002; 36: 20-42.

12 Legssyer R, Huaux F, Lebacq J, et al. Azithromycin reduces spontaneous and induced inflammation in F508del cystic fibrosis mice. Respir Res 2006; 7: 134.

13 Leal T, Lebacq J, Vanbinst R, et al. Successful protocol of anaesthesia for measuring transepithelial nasal potential difference in spontaneously breathing mice. Lab Anim 2006; 40: 43-52.

14 Lubamba B, Lebacq J, Lebecque P, et al. Airway delivery of low dose miglustat normalizes nasal potential differenece in F508del cystic fibrosis mice. Am J Respir Crit Care Med 2009; 179: 1022-1028.

15 Gizurarson S. Animal models for intranasal drug delivery studies. A review article. Acta Pharm Nord 1990; 2: 105-122.

16 Leal $\mathrm{T}$, Lebacq J, Lebecque $\mathrm{P}$, et al. Modified method to measure nasal potential differenece. Clin Chem Lab Med 2003; 41: 61-67.

17 Leal T, Fajac I, Wallace HL, et al. Airway ion transport impacts on disease presentation and severity in cystic fibrosis. Clin Biochem 2008; 41: 764-772.

18 Phalen RF, Mannix RC, Drew RT. Inhalation exposure methodology. Environ Health Perspect 1984; 56: 23-34.

19 Raeburn D, Underwood SL, Vilamil ME. Techniques for drug delivery to the airways, and the assessment of lung function in animal models. J Pharmacol Toxicol Methods 1992; 27: 143-159.

20 Cryan SA, Sivadas N, Garcia-Contreras L. In vivo animal models for drug delivery across the lung mucosa barrier. Adv Drug Deliv Rev 2007; 59: 1133-1151.

21 Grubb BR, Rogers TD, Boucher RC, et al. Ion transport across CF and normal murine olfactory and ciliated epithelium. Am J Physiol Cell Physiol 2009; 296: C1301-C1309.

22 Mery S, Gross EA, Joyner DR, et al. Nasal diagrams: a tool for recording the distribution of nasal lesions in rats and mice. Toxicol Pathol 1994; 22: 353-372. 
23 Harkema JR, Carey SA, Wagner JG. The nose revisited: a brief review of the comparative structure, function, and toxicologic pathology of the nasal epithelium. Toxicol Pathol 2006; 34: 252-269.

24 Dormer RL, Harris CM, Clark Z, et al. Sildenafil (Viagra) corrects F508del-CFTR location in nasal epithelial cells from patients with cystic fibrosis. Thorax 2005; 60: 55-59.

25 Berry B, Altman P, Rowe J, et al. Comparison of pharmacokinetics of vardenafil administered using an ultrasonic nebulizer for inhalation versus a single 10-mg oral tablet. J Sex Med 2009; [Epub ahead of print DOI: 10.1111/j.1743-6109.2009.01403.x].

26 Montgomery GS, Sagel SD, Taylor AL, et al. Effects of sildenafil on pulmonary hypertension and exercise tolerance in severe cystic fibrosis-related lung disease. Pediatr Pulmonol 2006; 41: 383-385.
27 Ionescu AA, Ionescu AA, Payne $\mathrm{N}$, et al. Subclinical right ventricular dysfunction in cystic fibrosis: a study using tissue Doppler echocardiography. Am J Respir Crit Care Med 2001; 163: 1212-1218.

28 Poschet J, Perkett E, Deretic V. Hyperacidification in cystic fibrosis: links with lung disease and new prospects for treatment. Trends Mol Med 2002; 8: 512-519.

29 Toward TJ, Smith N, Broadley KJ. Effect of phosphodiesterase-5 inhibitor, sildenafil (Viagra), in animal models of airways disease. Am J Respir Crit Care Med 2004; 169: 227-234.

30 Poschet JF, Timmins GS, Taylor-Cousar JL, et al. Pharmacological modulation of cGMP levels by phosphodiesterase 5 inhibitors as a therapeutic strategy for treatment of respiratory pathology in cystic fibrosis. Am J Physiol Lung Cell Mol Physiol 2007; 293: L712-L719. 